\title{
Impact of additional duodenojejunosotmy- site colo- nopexy on reduction delayed gastric emptying following pylorus-preserving pancreaticoduodenectomy: A pro- spective, randomized controlled study
}

\author{
Ho Kyoung HWANG, Chang Moo KANG*
}

Department of HBP Surgery, Yonsei University College of Medicine, Seoul, Korea

Introduction: Delayed gastric emptying (DGE) is a major complication after pylorus-preserving pancreaticoduodenectomy (PPPD). Although various hypotheses have been addressed as a cause of DGE following PPPD, the exact mechanism is still unknown. Gastric outlet angle resulted from retraction of duodenojejunostomy (DJ) site toward pancreaticojejunostomy (PJ) site during wound healing process of PJ can lead to mechanical disturbance of ingested food passage. Therefore, additional DJ-colon pexy procedure may reduce the incidence of DGE following PD.

Methods: From February 2016 to March 2019, 78 patients were recruited for this randomized controlled trial. The incidence of DGE was analyzed according to whether or not DJ-colon pexy was used after PPPD.

Results: PPPD without DJ-colon pexy (No DJ-pexy group) was performed in 39 patients, and 39 patients underwent PPPD with DJ-colon pexy (DJ-pexy group). A comparative analysis between the two groups showed no differences in age, sex, diagnosis, operation time, hospital stay, or postoperative complications, including pancreatic fistula $(p>0.05)$. Overall, DGE developed in seven patients $(17.9 \%)$ in the No DJ-pexy group and in 10 patients $(25.6 \%)$ in the DJ-pexy group $(p=0.411)$. The clinically relevant DGE (grades B and C) was also similar between two groups $(12.8 \%$ vs. $17.9 \%, p=0.530)$. The day that patients could eat more than half of the solid oral intake amount was marginally earlier in the Braun group (8.7 vs. 12.4 days, $p=0.054$ ).

Conclusions: The incidence and severity of DGE did not differ between DJ-pexy and No DJ-pexy group after PPPD. 